\title{
Development Status and Prospect of Minimum Quantity Lubrication Cutting Techonology
}

\author{
Xiufang Bai, Juan Jiang \\ Mechanical and Electrical Engineering Department, Qingdao Binhai Universtiy, Qingdao, \\ China,266555 \\ bxf1979@126.com,jj5302@126.com
}

\begin{abstract}
Keywords: Minimum quantity lubrication; Green machining; Cutting techonology; Nanofluid
\end{abstract}
\begin{abstract}
Because of significantly small amount of cutting fluid and vegetable oil with degradation function used in minimum quantity lubrication system, the environment is greatly improved. At the same time, the introduction of cutting fluid greatly improves the cooling and lubrication conditions in the cutting process, which not only reduces the cutting temperature and force, but also reduces the wear of the tool. Therefore, minimum quantity lubrication technology has been widely used. The technology is briefly introduced, as well as its development and research status. Among them, the Nanofluid minimum quantity lubrication has been paid more and more attention by domestic and foreign scholars due to its remarkable effect of cooling and lubrication. Finally, the future research direction is put forwards.
\end{abstract}

\section{Introduction}

In traditional machining, a large amount of cutting fluid is usually poured into the cutting area to reduce the cutting temperature. Cutting fluid has a certain effect on cooling, lubrication, rust prevention, chip removal and so on. Besides, it can improve the surface quality of the workpiece, the tool life and the processing efficiency. However, the use of cutting fluid not only greatly increases the cost of production, but also causes serious pollution on the environment, as well as harming the health of the human body.

In order to protect the environment and save the cost of purchasing cutting fluid and disposal of waste liquid, dry cutting technology without cutting fluid has been developed. For abandoning the cutting fluid in dry machining, the production cost and the environmental pollution is reduced greatly so that working environment and job satisfaction is significantly improved. A quantity of heat is produced in dry machining without the use of cutting fluid, which deteriorates the surface quality, increase the surface roughness, shortens the tool life, and the chip is easy to stick on the surface of the workpiece. On the premise of ensuring the lubrication performance and cooling effect , minimum quantity lubrication(MQL) emerges with using minimal amount of cutting fluid.

\section{Brief Introduction of MQL}

MQL is a new type of green machining technology, which is mixed with a small amount of lubricant in high pressure air and pressed into the cutting zone with high temperature[1]. High pressure air flow plays the role of cooling and chip removal. The lubricating fluid is adhered to the surface of the workpiece, forming a protective film, which plays the role of lubrication. This technology combines the advantages of spurring and dry machining, and the lubrication effect is almost same with the traditional. Vegetable oil with excellent biodegradability is generally used as the base oil[2], which has good lubrication performance, high viscosity index, low volatility, short production cycle, less environmental pollution, etc. Compared with traditional process, the amount of lubricant is only one part in ten thousands[3], which eliminates the pollution caused by large amount use of cutting fluid, and improves the working environment greatly. In addition, the introduction of lubricants medium greatly improves the cooling and lubrication conditions in the cutting process with respect to dry cutting, reducing the cutting temperature and force as well as the wear of the tool[4]. 


\section{Research Status of MQL}

In MQL system, compressed gas and vegetable oil are sprayed to the cutting area after mixing. The cooling is achieved through heat convection and evaporation of the mist particles. But little heat of vaporization and low heat transfer coefficient cause poor cooling effect, especially when in high temperature the lubrication ability of vegetable oil will decrease greatly. Therefore, it is urgent to find a new cooling lubrication medium to improve the lubricating and cooling capacity of MQL.

Cryogenic and Minimal Lubrication. Cryogenic and minimal lubrication is a kind of near dry cutting technology, which is used to inject mixture of the low temperature compressed air (usually $-10-30^{\circ} \mathrm{C}$ ) and minimal lubricant into the cutting zone, and to cool and lubricate it. Cooling medium can be cold air, liquid nitrogen, carbon dioxide and hydrogen, etc.. The low temperature cooling medium is ejected from the nozzle to the cutting zone by the cold air generator. At present, the research on the cryogenic and minimal lubrication technology is mainly focused on the manufacture of cryogenic and minimal lubrication supply device and application effect in cutting process. Compared with dry cutting, the cutting force and cutting temperature and so on are obviously reduced, and the surface quality of the workpiece is improved. At the same time, the tool wear is delayed and the machining efficiency is improved. However, a large amount of cryogenic medium is needed to complete the process, and the cost of preparation, storage and transportation is high. Besides, it is only suitable for small cutting force, so it is restricted in industrial production.

Nanofluid Minimal Quantity Lubrication. According to the theory of heat transfer, the heat transfer capacity of solid is higher than that of liquid, and the liquid higher than the gas. Based on the above, a certain amount of nano solid particles are added to the biodegradable lubricant to form nano fluid, which is atomized in high pressure and sprayed into the cutting zone. The nanoparticles increase the heat transfer capacity of the cutting fluid and play the role of cooling. In addition, nano particles have such tribological properties as special anti-wear and friction reduction and high load capacity in lubrication and tribology [5]. Nano fluid MQL not only has the advantages of MQL, but also strengthens the cutting heat transfer, which significantly improves the surface quality of the workpiece and reduces burn phenomenon, as well as improving the tool life and the working environment. Therefore, it is a kind of cutting technology with friendly ecology, low consumption and high efficiency.

Research Status at Home and Abroad. The excellent ability of nanofluids to enhance the cooling performance has attracted the attention of researchers at home and abroad. At present, domestic and foreign scholars have made some progress in the addition of nano particles in MQL technology. B. Shen [6]studied grinding performance by adding MoS2 into paraffin and soybean oil. The results indicated that, the paraffin-MoS2 nanofluid showed the benefits of reducing friction force and grinding force, compared with the base oil without nano particles. P. Kalita[7] evaluated grinding performance of MQL using AlS2 nanoparticles based on soybean oil, in comparison to dry grinding, flood lubriction and pure MQL grinding with soybean oil. The results showed that MQL with nanofluids had reduced friction coefficient, better surface quality of workpiece and less wheel wear. Lee[8] and Prabhu [9] studied the micro grinding with nanofluid MQL. The results reported that grinding force was reduced and the surface quality was improved. Liao[10]carried out a series of grinding experiments with MQL and nanofluid MQL. The experiments obtained smaller grinding force and friction coefficient. Kalita [11] performed the experiment with nanofluid in terms of grinding energy and friction coefficient. It was reported that the result showed reduced power consumption and wheel wear and improved surface finish. After adding nano particles, the cooling and lubrication effect was greatly improved on the interface between the wheel and the workpiece.

Jung[12] conducted a series of micro-drilling experiments in the cases of compressed air lubrication, pure MQL and nanofluid MQL. For nanofluid MQL, nano-diamond particles having the diameter of $30 \mathrm{~nm}$ were used with the base fluids of paraffin and vegetable oils. The experimental results showed that nanofluid MQL significantly increased the number of drilled holes and reduced the drilling torques and thrust forces. In addition, nanofluid MQL effectively eliminated remaining chips and burrs to enhance the quality of drilled holes. Marcon[13] formed nanographite fluid in 
MQL milling. The results showed that nanofluid MQL, relative to dry cutting, significantly reduced the cutting force, improved the surface quality, and the lower the particle concentration and nano fluid flow were, the smaller the cutting force.

Paturi[14]added WS2 nanoparticles to form nanofluids in MQL turning experiment. Through the analysis of variance and multivariate linear regression, the optimal value of cutting speed, feed rate, cutting depth was obtained which influences surface roughness. Experimental results showed that the finish quality of machined surface with nanofluid MQL imcreased by $35 \%$ compared with pure MQL.

Mao[15]performed the surface grinding experiments with nanofluid MQL method under different nanofluid parameters and reported that nanofluid MQL grinding with higher concentration nanoparticle generated lower grinding force, grinding temperature and surface roughness than that with lower concentration nanoparticle. In addition, increase in diameter of nanoparticle led to slight reduction in tangential grinding force and worse surface finish. $\mathrm{Su}$ [16]investigated the effect of nanofluid MQL with vegetable-based oil and ester oil as base fluids on cutting force and temperature in cylindrical turning of AISI 1045 medium carbon steel. Comparative experiments were carried out under different cooling/lubrication conditions, i.e., dry cutting, pure MQL with vegetable-based oil and unsaturated polyol ester, nanographite MQL. The experimental results showed that application of graphite oil-based nanofluid MQL reduced the cutting force and temperature significant1y. Furthermore, nanographite MQL with vegetable-based oil showed better performance than nanographite MQL with ester oil in terms of reduction in cutting force and temperature, especially at a high cutting speed.

Professor Li and his research team in Qingdao university of science and technology carried on deep research of nanoparticle jet MQL in grinding. The study focused on plane grinding heat transfer mechanism and surface integrity assessment, theoretical modeling and experimental research of three-dimensional velocity field and pressure field in wheel/workpiece wedge space, and the performance evaluation of different nanofluids lubricant and vegetable oil as base oil $[17,18]$.

Based on theory of heat transfer enhancement, the heat exchange capability of solid exceeds that of liquid and gas. Based on this feature, a certain quantity of nanolevel solid particles is added to degradable oil to form a nanofluid, which is atomized with high-pressure gas and sent to the grinding zone in the form of jet flow. This technology can not only solve the disadvantage of MQL in cooling effect, greatly improve the machining environment, save energy and reduce the cost to implement green manufacturing, but also make cutting fluid more effective to go through gas barrier into the cutting zone so as to improve manufacture precision of workpiece. In addition, due to special lubrication and tribological properties of nanoparticle, nanolevel shear film is formed in the tool/workpiece interface to further improve the lubrication performance, in turn improve tribological properties, which reduces the cutting force and cutting specific energy to lower the temperature of cutting zone further. Therefore ,the applications of nano particles to MQL cutting will greatly improve the effect of cooling and lubrication, making cutting high efficiency, low consumption, clean and low-carbon.

\section{Summary}

With the rapid development of nanomaterials science, the scholars at home and abroad have performed a large quantity of researches in respect to adding nanoparticles into the liquid to improve its coefficient of thermal conductivity and applying to the grinding, milling, drilling, and turning. However, most of the studies are limited to such comparison between MQL and traditional cooling methods as surface roughness, cutting force, cutting temperature etc. While the internal nature of convection heat transfer, the size effect, surface effect and the interface coupling effect of nanoparticles, how to form the shear film in the cutting zone, how to improve the lubrication and tribology performance using friction, deformation of nanoparticles collision, squeezing and roller, all these above are not in-depth researched. In order to argument the feasibility of nanofluid as a new technology of green cutting method, reveal the scientific nature for strengthening heat and 
tool/workpiece interface tribological characteristics, and provide a solid technology foundation for the practical application, it is necessary to explore scientific problems and key techniques in theory and experiment research.

\section{References}

[1] U Heisel, D Lutz, R Wassmer and U Walter: The minimum quantity lubricant technique and its application in the cutting process. Machines and Metals Magazine, Vo1.386(1998), p.22.

[2] U Krahenbuhl: Vegetable oil-based coolants improve cutting performance. Tooling \& production, Vol.68 (2002), p.34.

[3] J.P Davim, P.S Sreejith, R. Gomes and C Peixoto: Experimental studies on drilling of aluminum (AA1050) under dry, minimum quantity of lubricant, and flood-lubricated conditions. Journal of Engineering Manufacture,Vol.220(2006) No.10, p.1605.

[4] A Attanas, M Ge1fi, C Giardini and C Remino: Minimal quantity lubrication in turning:effect on tool wear. Wear, Vol.260(2006) No.3, p.333.

[5] C Choi, M. Jung, Y Choi, J Lee and J Oh: Tribological Properties of Lubricating Oil-Based Nanofluids with Metal/Carbon Nanoparticles. Journal of Nanoscience and Nanotechnology, (2011) No.11, p.368.

[6] B Shen, A.P Malshe, P Kalita and A.J Shih: Performance of novel MoSa nanoparticles based grinding fluids in minimum quantity lubrication grinding. Transactions of NAMRI/SME, Vol.36(2008), p.357.

[7] P Kalita, A.P Malshe, W Jiang and A.J.S Nanomech: Tribological Study of Nano Lubricant Integrated Soybean Oil for Minimum Quantity Lubrication (MQL) Grinding authors. Transaction of NAMRI/SME,Vol.38(2010), p.137.

[8] P.H Lee, J.S Nam, C Li and S.W Lee: An experimental study on micro-grinding process with nanofluid minimum quantity lubrication (MQL) .International Journal of Precision Engineering and Manufacturing, Vol.13(2012) No.3, p.331.

[9] S Prabhu and B.K Vinayagam: Nano surface generation of grinding process using carbon nano tubes. Sadhana,Vol.35(2010) No.6, p.747.

[10]Y.S Liao, Y.P Yu and C.H Chang: Effects of Cutting Fluid with Nano Particles on the Grinding of Titanium Alloys. Advanced Materials Research, Vol.126(2010), p.353.

[11]P Kalita, A.P Malshe and K.P. Rajurkar: Study of tribo-chemical lubricant film formation during application of nanolubricants in minimum quantity lubrication (MQL) grinding. CIRPAnnals-Manufacturing Technology, Vol.61(2012) No.1, p.327.

[12]S.N Jung, P.H Lee and S.W Lee: Experimental characterization of micro-drilling process using nanofluid minimum quantity lubrication. International Journal of Machine Tools\& Manufacture, Vol.51(2011), p.649.

[13]A Marcon, S Melkote, K Kalaitzidou and D DeBra: An experimental evaluation of graphite nanoplatelet based lubricant in micro-milling. CIRP Annals-Manufacturing Technology, Vol.59(2010), p.141.

[14]U.M.A Paturi, Y.R. Maddu, R.R Marur and S.K.R Narala: Measurement and analysis of surface roughness in WS2 solid lubricant assisted minimum quantity lubrication(MQL)turning of Inconel 718.Procedia CIRP,Vol.40(2016), p.138.

[15]C Mao, X.J Tang, H.F Zou, X.M Huang and Z.X Zhou: Investigation of grinding characteristic using nanofluid minimum quantity lubrication. International Journal of Precision Engineering and Manufacturing, Vol.13(2012) No.10, p.1745.

[16]Y. Su, L. Gong, Z.Q Liu and D.D. Chen: Performance evaluation of nanofluid MQL with vegetable-based oil and ester oil as base fluids in turning. Int $\mathrm{J}$ Adv Manuf Technol,Vol.83(2016), p.2083.

[17]C.H Li, Z.R Liu, W.P Mao and G.Q Cai: Investigation of Coolant Fluid through Grinding Zone in High-Speed Precision Grinding. Journal of Donghua University, Vol.27(2010), No.1, p.87. 
[18]Y.G Wang, C.H Li and Y.B Zhang; Experimental evaluation of the lubrication properties of the wheel/work piece interface in MQL grinding with different nanofluids. Tribology International, Vol.99(2016), p.198. 